\title{
Me, My Cell, and I: The Role of the Collision Cell in the Tandem Mass Spectrometry of Macromolecules
}

\author{
Margaret G. McCammon and Carol V. Robinson \\ Cambridge University Chemical Laboratory, Cambridge University, Cambridge, UK
}

\section{Introduction}

The molecular apparatus of the cell comprises a multitude of large protein assemblies that carry out the majority of biological activities. These cellular machines are often heterogeneous in nature, with transient noncovalent interactions playing a vital role in their function. Such dynamic characteristics make investigation by traditional structural biology approaches challenging, but in recent years mass spectrometry (MS), a relative newcomer to the field, has developed at a spectacular pace to offer a novel molecular viewpoint. Today MS provides far more than simple mass measurement, contributing in multiple ways to our understanding of these cellular assemblies. Much of this new ability is attributable to the flexible application of collisioninduced dissociation (CID).

The role of collision gas, both in the collision cell and out of it, is crucial for the application of MS to problem solving in complex biological systems. Balancing instrument pressures with collision gas facilitates the transfer of intricate biological complexes into the gas phase for analysis while intact, as opposed to measuring individual dissociated subunits. Careful manipulation of these pressures can disrupt solution-derived buffer ions, without dissociating the high mass complex. This, in turn, maximizes the resolution of the mass spectrum to reveal structural detail within the macromolecule. In this way MS provides a superb complement to more established approaches, such as nuclear magnetic resonance (NMR), in which the upper mass limit for investigation of similarly heterogeneous complexes is approximately 100 $\mathrm{kDa}$. For approaches that can be applied to complexes of more than $100 \mathrm{kDa}$, such as ultracentrifugation or chromatography, the constraint in resolution limits the level of structural detail that can be elucidated. By contrast, MS can be applied to the detailed analyses of multimeric macromolecules in the megadalton range.

The objective of this article is to provide an overview of the techniques and instrumentation involved in this approach and attempt to convey a measure of the potential of this rapidly evolving field. Through this, we hope to give a personal view as to why we believe high mass MS to be one of the most intriguing and exciting of the new biophysical approaches.

\section{In the Beginning, There Was Atom}

The development of two components in particular, the ionization source and the mass analyzer, have played a pivotal role in the ascent of biological MS. To truly demonstrate the remarkable attributes of this field, we must begin where this process begins; namely, sample ionization.
Traditionally, the strength of MS was in its ability to distinguish differences in atomic mass, such as in isotope mixtures. Innovative research during the 1960s and 1970s lent MS a new biological dimension and led to the characterization of many natural products. In the 1980s, the somewhat eccentric coupling of MS with electrospray, an industrial process for spray painting cars, resulted in the development of electrospray ionization (ESI), culminating in the first ever mass spectrum of an intact protein in 1989 (1). Over time, this process was refined, and in 2002, these endeavors were rewarded with a share of the Nobel Prize in Chemistry.

In our research we use nano-ESI, a process by which an electric field is imposed onto a gold-plated borosilicate needle containing the solution to be analyzed (Figure 1A). This creates an electric stress that causes the analyte liquid to develop an electric double layer, leading to charge accumulation at the liquid surface. As a result the solution meniscus is destabilized, such that a jet of droplets with an excess of positive (or negative) ions is formed at the end of the capillary in the so-called Taylor cone. Thus, the liquid is converted into a fine mist of droplets with excess charge. As the solvent evaporates, this charge is passed onto the naked gas phase ion, and the different numbers of charge per molecule give rise to a distribution of charged variants for each species. An important advantage of ESI-MS in biological studies is that this process can be applied to aqueous buffer solutions at neutral $\mathrm{pH}$.

Once in the gas phase, noncovalent complexes are analyzed primarily by the time-of-flight (TOF) mass analyzer. Here, the ions are accelerated through a fixed potential into a field-free drift region. Low mass ions achieve higher velocity than those of high mass, and since they have a fixed kinetic energy, the exact mass of an ion can be deduced from the amount of time it takes to reach the detector. The data are represented as a mass spectrum, which records the mass-to-charge ratio $(\mathrm{m} / \mathrm{z})$ of the ion.
A
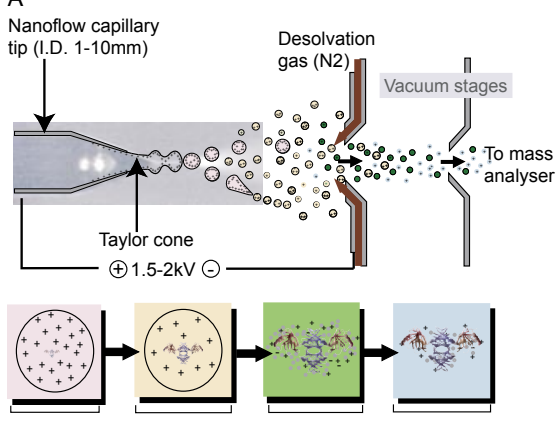

lon desolvation

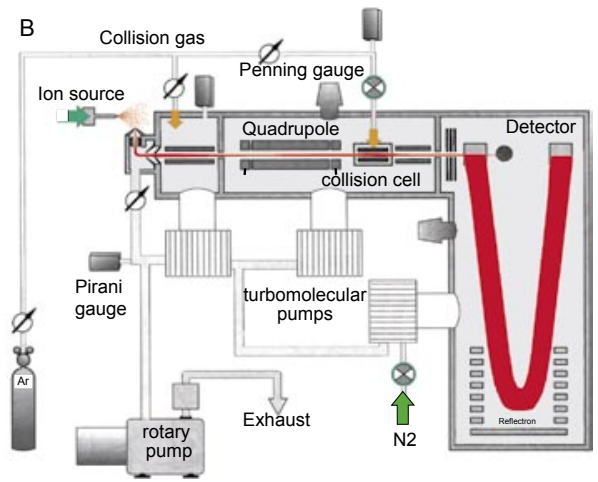

Figure 1. Representation of nano-ESI and the tandem MS process. (A) During nano-ESI the Taylor cone elongates into a filament that breaks into droplets. Solvent evaporates from the initial droplet (pink) to progressively smaller droplets to leave a single, multiply charged molecule (blue). (B) Representation of the modified high mass Q-TOF2 instrument. The analyte is sampled into vacuum through the ion source (green) to the ion path (red). Features used for pressure adjustment are described in detail elsewhere (2). ESI, electrospray ionization; MS, mass spectrometry; TOF, time-of-flight. 


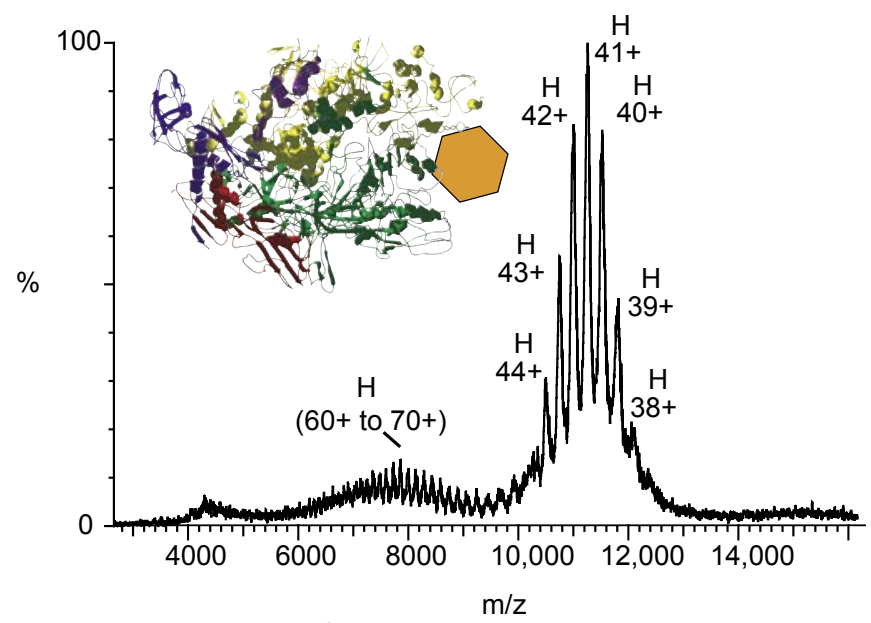

Figure 2. Mass spectrum of the intact Escherichia coli RNA polymerase holo complex $(\mathrm{H})$ at $\mathrm{m} / \mathbf{z} \mathbf{1 0 , 0 0 0 - 1 2 , 0 0 0}$. The charge state envelope at lower intensity $(6000-10,000 \mathrm{~m} / \mathrm{z})$ is assigned to a complex of identical composition with increased subunit flexibility. (Inset) X-ray crystal structure of the complex comprising $\alpha_{2}$ (red and blue), $\beta$ (green), $\beta^{\prime}$ (yellow), $\Omega$ (purple) with a schematic representation of $\sigma^{70}$ (orange) (10). $\mathrm{m} / \mathrm{z}$, mass-to-charge ratio.
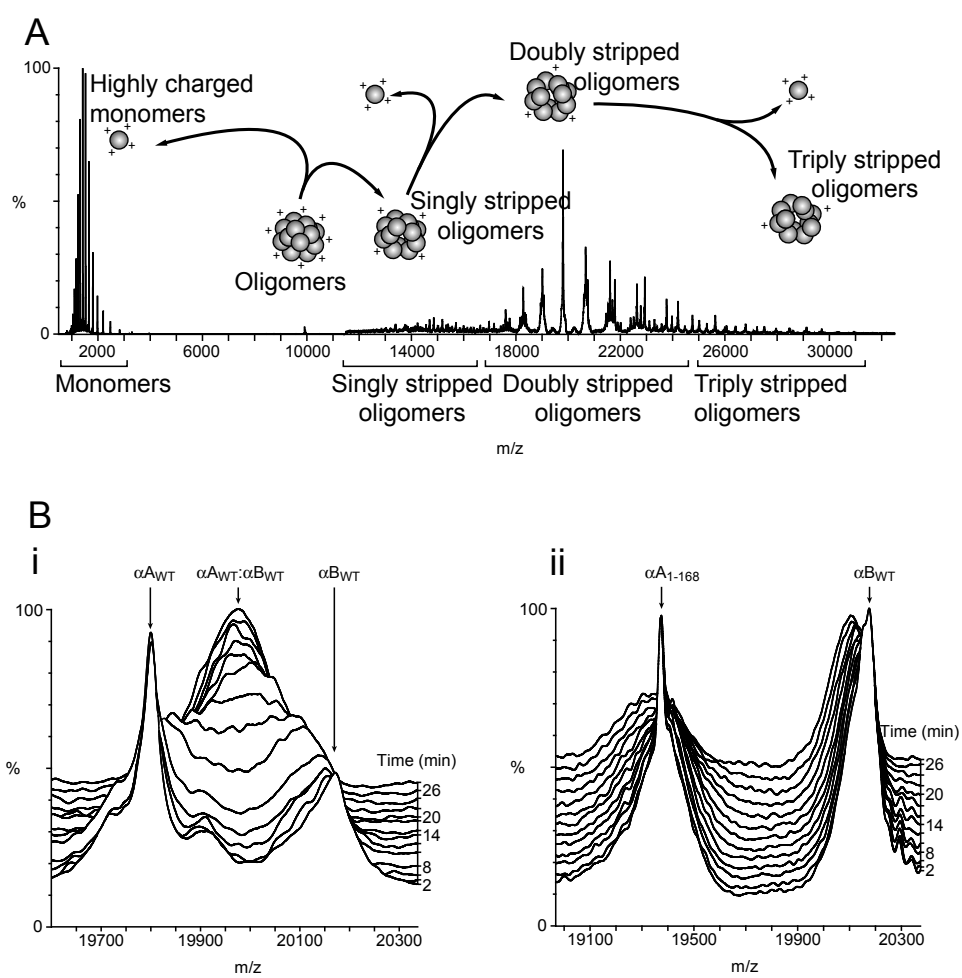

Figure 3. MS analysis of subunit dynamics. (A) Tandem MS and CID of oligomeric $\alpha A$ crystallin, resulting in dissociation of the oligomers into monomers at low $\mathrm{m} / \mathrm{z}$ and stripped oligomers at high $\mathrm{m} / \mathrm{z}$. (B) The effect of truncation on subunit exchange. (panel B, i) Over a 30-min period $\alpha A$-crystallin and $\alpha B$-crystallin homotetramers are diminished, to be replaced with a peak corresponding to hetero-oligomers of the two subunits. (panel $B$, ii) $\alpha A_{1-168}$-crystallin and $\alpha B$-crystallin over the same time-course in which subunit exchange is not observed. MS, mass spectrometry; CID, collision-induced dissociation; $\mathrm{m} / \mathrm{z}$, mass-tocharge ratio.
Since the ESI process leads to multiple charging of each species, a series of peaks with different $\mathrm{m} / \mathrm{z}$ values, corresponding to the same molecular mass, is observed in what is known as a charge state envelope. Solving a series of equations for the charge state envelope reveals the mass of the species.

To make this approach amenable to the investigation of heterogeneous macromolecules, we developed a tandem mass spectrometer customized for the optimal transmission of high mass assemblies (2). This formed the basis of our subsequent instrumentation. Figure 1B shows our typical experimental setup where, in addition to the collision cell, gas inlets in the ion source and ion guide stage facilitate the control of pressure levels throughout the instrument. This capability is essential for the transmission of high mass entities. Another important feature is the low-frequency quadrupole, which enables us to isolate individual charge states of up to $32,000 \mathrm{~m} / \mathrm{z}$ from the population of species. The isolated species can then be injected into the argon atmosphere of a dedicated collision cell for CID, and ions dissociated individually in a form of two-dimensional gas phase analysis known as tandem MS.

\section{Measuring Up}

Biological assemblies of increasingly high mass are now being analyzed by MS, with multiple low energy collisions playing a central role in preserving their weak intermolecular interactions. While not CID in its conventional sense, the importance of this aspect is such that it would be remiss not to cover it in this essay. This import is because during the transition from solution to gas phase (and therefore from atmospheric pressure to vacuum), the ESI stream containing the complex ions expands adiabatically, and consequently, the ions experience a destabilizing gain in internal energy. This is offset by introducing low levels of collision gas at multiple points throughout the ion flight path to activate the ion in a process known as collisional cooling. Instead of increasing the rate of dissociation, as might intuitively be expected, collisions with the excess gas molecules offer the complex ions a means of dampening their translational energy and thereby facilitate transmission of high mass complexes through to the high vacuum of the TOF mass analyzer.

Conveying intact macromolecules into the gas phase has limited application however, if the stoichiometry of interacting components cannot be distinguished due to low resolution on the mass spectrum. Inefficient removal of water and buffer molecules, which remain attached during the transition from solution phase, gives rise to broad charge states in the mass spectrum with an associated reduction in mass measurement accuracy. This can be corrected by optimizing the input of CID energy so the ions are "heated," such that the majority of water and buffer molecules are stripped away without destroying the weak noncovalent interactions within the complex. Direct mass measurement of this resolved complex can then reveal its subunit make up and, often, details of its component architecture.

These processes, dampening translational energy and CID of nonspecific buffer molecules, have been exploited to provide new information on the multicomponent holoenzyme core of RNA polymerase from Escherichia coli (3). Using collision gas to balance the pressures within the mass spectrometer, it was possible to observe the intact core enzyme bound to a $\sigma$ factor $\left(\alpha_{2} \beta \beta^{\prime} \Omega \sigma^{70}\right)$, a complex comprising six individual subunits with an overall mass in excess of $460 \mathrm{kDa}$ (Figure 2). This is an interesting example, 
because it highlights several other important features of high mass MS. In this case, the core enzyme had been isolated from natural sources and was not subjected to enrichment strategies. This compares to the more usual source of protein complexes produced by recombinant overexpression followed by reconstitution of individual subunits. Detailed analysis of such a minute amount of sample was possible only because nano-ESI MS can be carried out on microliter quantities of micromolar solutions.

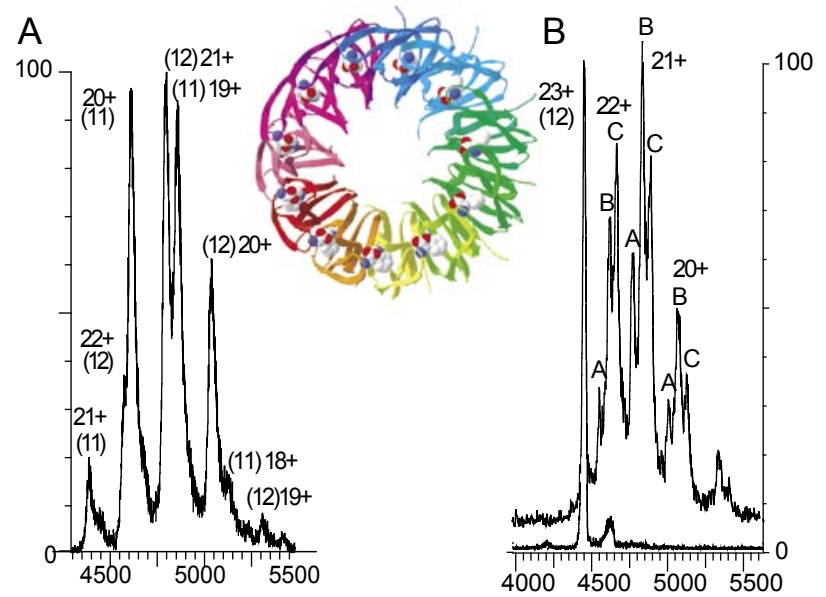

Figure 4. MS analysis of liquid binding in macromolecules. (A) Mass spectrum of the TRAP:trp complex showing two populations corresponding to TRAP 11 and TRAP 12 associated with 11 tryptophan molecules. (B) Tandem MS of the TRAP 123 + charge state showing subpopulations of apo TRAP (peaks labeled A) and TRAP ${ }_{12}$ with 6 tryptophan molecules (peaks labeled B) and TRAP $_{12}$ with 11 tryptophan molecules (peaks labeled C). (Inset) Crystal structure of the TRAP 1 : $\operatorname{trp}_{11}$ adapted from Protein Data Bank (pdb) code 1c9s with subunits in different colors and tryptophan as CPK (Corey, Pauling, and Koltan). TRAP, tryptophan RNA-binding protein; trp, tryptophan; MS, mass spectrometry.

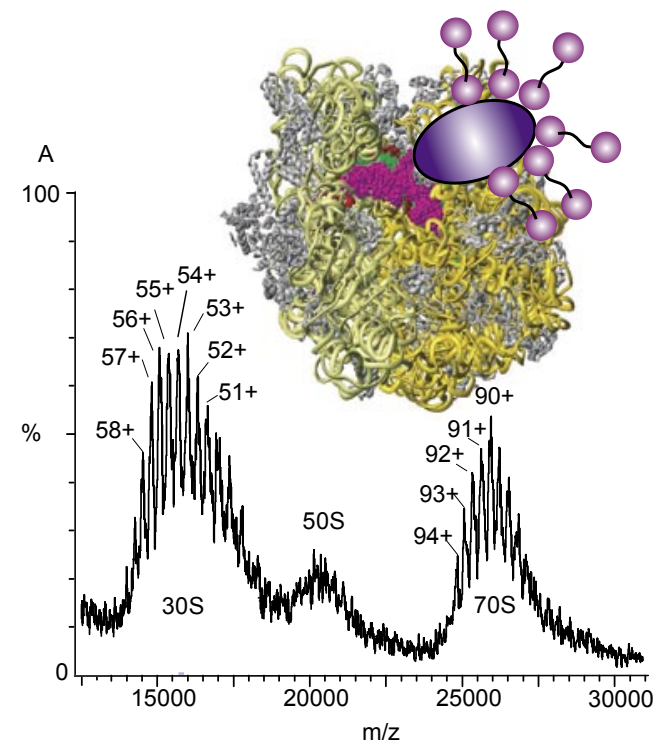

B

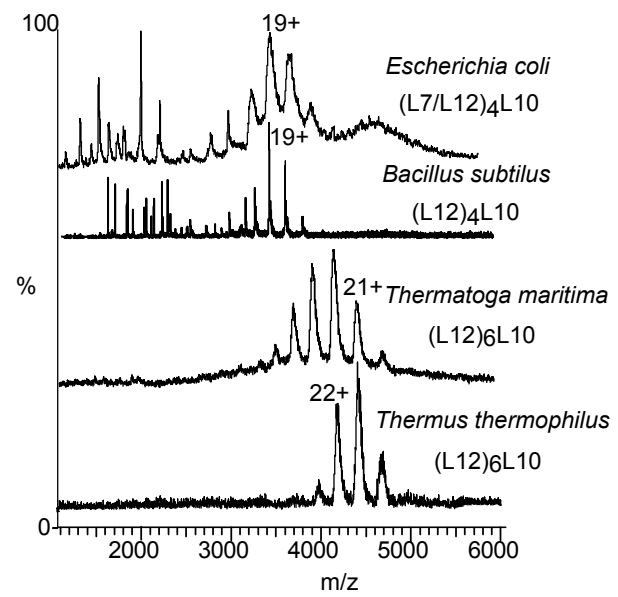

Figure 5. Probing the architecture of the ribosome. (A) Mass spectrum showing resolved charge states for the 70S ribosome of Thermus thermophilus with dissociated $50 \mathrm{~S}$ and $30 \mathrm{~S}$ subunits. (Inset) X-ray crystal structure of the 70S particle with ribosomal RNA (rRNA) shown in gold and yellow for the 50S and 30S subunits, respectively. Proteins in grey with a schematic of the stalk complex comprising L10 (blue) and six L12 (pink). Adapted from Protein Data Bank (pdb) code 1gix. (B) Stalk complexes released from different bacterial ribosomes consistent with heptameric and pentameric stoichiometries for thermophiles and mesophiles, respectively. $\mathrm{m} / \mathrm{z}$, mass-to-charge ratio.
Further, examination of the mass spectrum revealed the presence of two distinct charge state envelopes where both the major species at $\mathrm{m} / \mathrm{z} 10,000-12,000$ (charge state $38+$ to $44+$ ) and the minor species at $\mathrm{m} / \mathrm{z} 6000-10,000$ (charge state $60+$ to $70+$ ) correspond to identical masses. This is due to the increased level of charging experienced by subunits in which additional amino acids are exposed to the buffer while in solution. This characteristic, therefore, indicates the presence in solution of two different conformations of the same noncovalent assembly, the more highly charged of which has subunits with relatively increased flexibility. This is a common feature in MS of intact complexes and reflects the capacity of this approach to elucidate subtle subunit dynamics within heterogeneous systems.

MS also provides an incomparable tool for understanding more discrete dynamic processes. Recently, in the first investigation of its kind, the exceptional resolving power of ESI-MS was used to demonstrate the presence in solution of subunit exchange in small heat shock proteins (4). Taking this approach a step further led to an investigation of a solution-based mechanism for subunit exchange in $\alpha A$-crystallin, $\alpha B$-crystallin, and $\alpha A_{1-168}$ crystallin, a truncation associated with aging in the human eye lens (5). MS analysis revealed a previously unknown capacity for $\alpha A_{1-168}$ to dramatically compromise subunit dynamics (Figure 3 ). Moreover, this truncation was shown to be associated with reduced oligomer polydispersity when compared to the wildtype, coupled with a preference to form oligomers composed of an even number of subunits. Importantly, this information was only available because MS measures all the populations of monomeric and multimeric species present in solution, as opposed to providing a single average mass. Given that, unlike DNA, the information contained within protein systems is not static, the importance of characterizing dynamic mechanisms such as subunit exchange cannot be overstated. As such, it is likely that this approach will have important implications for similar research in the future.

\section{Multiple Choice}

The manipulation of ligand populations in multimeric assemblies is another area of intense interest. Binding of proteins and their ligands is crucial in many cellular processes, often representing important targets for drug design. MS occupies a unique niche for the investigation of subtle conformational changes that occur in response to ligand binding. Once the complex has been achieved intact in the gas phase, careful manipulation of the environment to which the complex ions are exposed results in the controlled dissociation of individual components. This can reveal the presence of small molecules and ligands within the context of macromolecular complexes that would otherwise be too complicated to analyze. Such an approach was used to investigate drug binding 
in a protein-micelle complex of EmrE from E. coli (6). Transfer of the intact EmrE-dodecylymaltoside (DDM) species into the gas phase presented a significant challenge in itself, as the stabilizing hydrophobic forces are compromised with the removal of water. Under these circumstances, applying collisional cooling to enhance resolution is difficult without causing extensive disruption of the micelles, a challenge overcome by tandem MS and CID of the nonresolved complex. This revealed the presence of EmrE and DDM and a cation substrate tetraphenyl phosphonium (TPP+). Moreover, the dissociation profile indicated that EmrE remains associated with DDM even after the release of TPP+, demonstrating that important information can be extracted even from complexes not best suited to gas phase ionization. In addition to revealing stoichiometry and ligand binding, MS can provide new insights into the quaternary structural arrangement of macromolecules, making it a powerful adjunct to existing structural biology techniques. Such an approach led to the identification of a two-stage dissociation pattern in noncovalently bound tryptophan molecules from the protein ring structure of tryptophan RNA-binding protein (TRAP) (7). Figure 4 shows the presence of three distinct populations assigned as apo

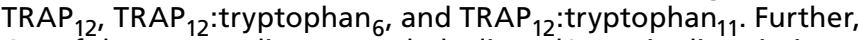
$C I D$ of the TRAP 12 dimer revealed a ligand/protein dissociation pattern that could correspond to only one of three possible structural arrangements, demonstrating the potential for MS in the elucidation of structural features of assemblies that are multiliganded.

\section{Under Pressure}

When the translational energy of the biomolecule is not dampened during phase transition, or if internal energy is increased by high energy collisions with residual gas molecules, the complex dissociates into its individual components. Controlling instrument pressures to effect dissociation of subunits can provide valuable information on intramolecular interactions and posttranslational modifications of large heterogeneous protein assemblies. In the investigation of one such particle, the Saccharomyces cerevisiae ribosome, CID dissociation was used to peel targeted components away from the macrostructure (8). These data revealed details in the composition of the proteins on the stalk complex that led to the definition of novel, low level posttranslational modifications. An analogous study of deletion variants also uncovered a cooperative process in the assembly of the stalk complex, involving binding of partner proteins to increase stability on the ribosome. This approach was taken further in a recent investigation of ribosomes from thermophilic bacteria in which complexes in excess of 2 megaDa were resolved in the mass spectrum (9). Through CID, phosphorylation was identified in a single component of this giant module, the stalk protein L12. Crucially, it was also demonstrated that this posttranslational modification is not present on the L12 during MS dissociation, but is only observed during tandem MS and CID of the intact $50 S$ component, where the stalk complex is situated. These data demonstrate, for the first time, that phosphorylation occurs exclusively while L12 is bound to the ribosome and suggests a role in anchoring the stalk to the ribosome.

The stalk complex has been studied intensively by biochemical means, and the pentameric stoichiometry identified in mesophiles was thought to be universal. In this study however, MS analysis of the mass of stalk complexes did not always conform to this stoichiometry, and using CID, the authors went on to demonstrate the presence of novel heptameric complexes from thermophilic bacteria (Figure 5). This is a particularly significant area for biological MS. The inherently dynamic nature of the ribosome stalk complex often makes it inaccessible to investigation by static approaches such as X-ray crystallography. In addition, the conformational heterogeneity of this feature can make it difficult to define by these approaches since, even in highly resolved structures, individual proteins cannot be discerned for this part of the ribosome. This MS approach is therefore proving to be an invaluable tool in defining dynamic complexes of this magnitude.

\section{A Flying Start}

Although still a relatively new technique, the rate of progress in MS from analysis at the atomic level to the study of complex biological systems has been breathtaking. Today, functional cellular particles, with molecular masses in the megadalton range, can be transferred intact into the gas phase. The plethora of new information gleaned from the rapidly improving resolution of these entities goes some way to explaining why MS is becoming increasingly popular for the investigation of heterogeneous supramolecular assemblies. The real-time nature of this approach provides an exclusive molecular vantage point for the interrogation of dynamic, noncovalent assemblies that cannot be analyzed by other means, shedding new light on the topology of high mass entities that are complicated by weak or fleeting interactions. With hindsight, the once seemingly insurmountable limitations of MS have proven to be merely transient setbacks, and we have every reason to believe the future holds equally spectacular innovations.

\section{References}

1. Fenn, J.B., M. Mann, C.K. Meng, S.F. Wong, and C.M. Whitehouse. 1989. Electrospray ionisation for mass-spectrometry of large biomolecules. Science 246:64-71.

2. Sobott, F., H. Hernandez, M.G. McCammon, M.A. Tito, and C.V. Robinson. 2002. A tandem mass spectrometer for improved transmission and analysis of large macromolecular assemblies. Anal. Chem. 74:14021407.

3. Ilag, L.L., L.F. Westbalde, C. Deshayes, A. Kolb, S.J.W. Busby, and C.V. Robinson. 2004. Mass spectrometry of Escherichia coli RNA polymerase: interactions of the core enzyme with sigma[70] and Rsd protein. Structure 12:269-275.

4. Sobott, F., J.L.P. Benesch, E. Vierling, and C.V. Robinson. 2002. Subunit exchange of multimeric protein complexes-real time monitoring of subunit exchange between small heat shock proteins by using electrospray mass spectrometry. J. Biol. Chem. 277:38921-38929.

5. Aquilina, J.A., J.L.P. Benesch, L.L. Ding, O. Yaron, J. Horwitz, and C.V. Robinson. 2005. Subunit exchange of polydisperse proteins: mass spectrometry reveals consequences of alpha A-crystallin truncation. J. Biol. Chem. 280:14485-14491.

6. Ilag, L.L., I. Ubarretxena-Belandia, C.G. Tate, and C.V. Robinson. 2004. Drug binding revealed by tandem mass spectrometry of a proteinmicelle complex. J. Am. Chem. Soc. 126:14362-14363.

7. McCammon, M.G., H. Hernandez, F. Sobott, and C.V. Robinson. 2004. Tandem mass spectrometry defines the stoichiometry and quaternary structural arrangement of tryptophan molecules in the multiprotein complex TRAP. J. Am. Chem. Soc. 126:5950-5951.

8. Hanson, C.L., H. Videler, C. Santos, J.P.G. Ballesta, and C.V. Robinson. 2004. Mass spectrometry of ribosomes from Saccharomyces cerevisiae-implications for assembly of the stalk complex. J. Biol. Chem 279:42750-42757.

9.Ilag, L.L., H. Videler, A.R. McKay, F. Sobott, P. Fucini, K.H. Nierhaus, and C.V. Robinson. 2005. Heptameric (L12)(6)/L10 rather than canonical pentameric complexes are found by tandem MS of intact ribosomes from thermophilic bacteria. Proc. Natl. Acad. Sci. USA 102:8192-8197.

10.Zhang, G.Y., E. Campbell, L. Minakhin, C. Richter, K. Severinov, and S.A. Darst. 1999. Crystal structure of Thermus aquaticus core RNA polymerase at 3.3 A resolution. Cell 98:811-824 